\title{
EDITORIAL
}

Multiple myeloma gammopathies

\section{On the road to molecular prognostication in SMM}

\author{
Salomon Manier ${ }^{1,2}$
}

Received: 2 June 2019 / Accepted: 15 August 2019 / Published online: 12 November 2019

C Springer Nature Limited 2019

Smoldering multiple myeloma (SMM) always precedes MM [1, 2]. However only $50 \%$ of patients will progress at 5 years and $66 \%$ at 10 years [3]. Therefore, defining high-risk SMM represents a clinically fundamental challenge in order to help treatment decision. Many different scoring systems have been developed to predict patients' outcome [4]. The most broadly used is the Mayo Clinic model that combines M-protein, the percentage of bone marrow plasma cells (BMPC\%), and the ratio of involved to uninvolved serum-free light chains (FLCr) [3]. The IMWG recently updated this model and defined new cutoff, namely $2 \mathrm{~g} / \mathrm{dL}$ for M-protein, $20 \%$ for BMPC\%, and 20 for the FLCr to categorize patients into three risk groups [5]. While this stratification model is essential for clinical trials it does not capture the molecular characteristics of the tumor cells and the role of the microenvironment, which are potentially crucial factors of progression in SMM.

In this issue, Drs Misund and Keane et al. report the role of MYC translocations in SMM progression (citation of the paper). They used a cohort of 23 MGUS samples, 90 SMM, and 612 newly diagnosed MM samples from the CoMMpass study. None of the MGUS samples had a MYC translocation but it was the case for 24 and $42 \%$ of SMM and MM samples, respectively. The time to progression was shorter in patients with SMM harboring an immunoglobulin (Ig)-MYC translocation, even after adjusting for the Mayo Clinic criteria. In contrast, non-Ig-MYC translocations did not correlate to shorter time to progression. Of note, DIS3 mutations were also associated with a higher risk of progression to $\mathrm{MM}$ in this cohort.

These data reinforce the fact that MYC translocation is one of the main secondary events in MM oncogenesis.

Salomon Manier

salomon.manier@chru-lille.fr

1 Department of Hematology, CHU Lille, University of Lille, Lille, France

2 INSERM UMR-S1172, Lille, France
Previous reports identified lower frequency of MYC translocations in newly diagnosed settings (18 and 23\%) [6, 7], compared with $42 \%$ in the present study. The type of algorithm used to call translocations can explain those differences, as well as the depth of sequencing and the size of the bait set covering the MYC locus in some case. In all analyses, one third of those MYC translocations involved an Ig partner $(I G H, I G K$, and $I G L)$ and two third a non-Ig partner. Most recurrent of the latter partners are $F A M 46 C$, FOXO3, BMP6, or TXNDC5, which are located at superenhancers sites in MM cells, resulting in $M Y C$ upregulation.

Interestingly, the authors observe different prognostic values of Ig-MYC vs. non-Ig-MYC translocations; only the first one being associated with adverse outcomes. It was recently reported that IgL-MYC translocations are a marker of poor prognosis in MM [7]. It has been suggested that IgL translocations induce IMiDs resistance by hijacking IKZFI. However the fact that Ig-MYC translocations are associated with a higher risk of progression from SMM to MM indicate an intrinsic high-risk property of those translocations, independently of any treatment. We can hypothesize that the mechanism underlying these translocations or simply the power of the Ig superenhancers can explain this phenotype.

While the present study might be limited by the number of SMM samples it is also a first step toward the molecular classification of SMM, in order to refine the risk stratification of those patients and help treatment decision. Another layer of understanding that future studies will probably elucidate is the role of the immune microenvironment and how it participates to the risk of progression in patients with SMM.

\section{Compliance with ethical standards}

Conflict of interest The author declares that he has no conflict of interest.

Publisher's note Springer Nature remains neutral with regard to jurisdictional claims in published maps and institutional affiliations. 


\section{References}

1. Landgren O, Kyle RA, Pfeiffer RM, Katzmann JA, Caporaso NE, Hayes RB, et al. Monoclonal gammopathy of undetermined significance (MGUS) consistently precedes multiple myeloma: a prospective study. Blood. 2009;113:5412-7.

2. Weiss BM, Abadie J, Verma P, Howard RS, Kuehl WM. A monoclonal gammopathy precedes multiple myeloma in most patients. Blood. 2009;113:5418-22.

3. Kyle RA, Remstein ED, Therneau TM, Dispenzieri A, Kurtin PJ, Hodnefield JM, et al. Clinical course and prognosis of smoldering (asymptomatic) multiple myeloma. New Engl J Med. 2007;356: 2582-90.
4. Rajkumar SV, Landgren O, Mateos MV. Smoldering multiple myeloma. Blood. 2015;125:3069-75.

5. Miguel JS, Mateos MV, Gonzalez V, Dimopoulos M, Kastritis E, Hajek R, et al. Updated risk stratification model for smoldering multiple myeloma (SMM) incorporating the revised IMWG diangostic criteria. ASCO. 2019.

6. Walker BA, Wardell CP, Murison A, Boyle EM, Begum DB, Dahir NM, et al. APOBEC family mutational signatures are associated with poor prognosis translocations in multiple myeloma. Nat Commun. 2015;6:6997.

7. Barwick BG, Neri P, Bahlis NJ, Nooka AK, Dhodapkar MV, Jaye DL, et al. Multiple myeloma immunoglobulin lambda translocations portend poor prognosis. Nat Commun. 2019;10:1911. 